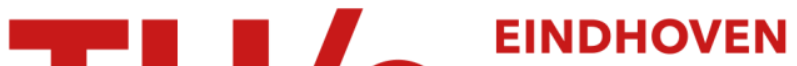 UNIVERSITY OF TECHNOLOGY
}

\section{Influence of geometry on acoustic end-corrections of slits in microslit absorbers}

\section{Citation for published version (APA):}

Aulitto, A., Hirschberg, A., \& Lopez Arteaga, I. (2021). Influence of geometry on acoustic end-corrections of slits in microslit absorbers. Journal of the Acoustical Society of America, 149(5), 3073-3085. [3073].

https://doi.org/10.1121/10.0004826

DOI:

$10.1121 / 10.0004826$

Document status and date:

Published: 06/05/2021

\section{Document Version:}

Accepted manuscript including changes made at the peer-review stage

\section{Please check the document version of this publication:}

- A submitted manuscript is the version of the article upon submission and before peer-review. There can be important differences between the submitted version and the official published version of record. People interested in the research are advised to contact the author for the final version of the publication, or visit the $\mathrm{DOI}$ to the publisher's website.

- The final author version and the galley proof are versions of the publication after peer review.

- The final published version features the final layout of the paper including the volume, issue and page numbers.

Link to publication

\section{General rights}

Copyright and moral rights for the publications made accessible in the public portal are retained by the authors and/or other copyright owners and it is a condition of accessing publications that users recognise and abide by the legal requirements associated with these rights.

- Users may download and print one copy of any publication from the public portal for the purpose of private study or research.

- You may not further distribute the material or use it for any profit-making activity or commercial gain

- You may freely distribute the URL identifying the publication in the public portal.

If the publication is distributed under the terms of Article 25fa of the Dutch Copyright Act, indicated by the "Taverne" license above, please follow below link for the End User Agreement:

www.tue.nl/taverne

Take down policy

If you believe that this document breaches copyright please contact us at:

openaccess@tue.nl

providing details and we will investigate your claim. 


\title{
Influence of geometry on acoustic end-corrections of slits in Microslit Absorbers
}

\author{
Alessia Aulitto ${ }^{\text {a) }}$ \\ Department of Mechanical Engineering Dynamics and Control, Eindhoven University of \\ Technology, P.O. Box 513, 5600 MB Eindhoven, The Netherlands \\ Avraham Hirschberg \\ Department of Applied Physics, Gas Dynamics and Aero-Acoustics, Eindhoven University \\ of Technology, P.O. Box 513, 5600 MB Eindhoven, The Netherlands \\ and Ines Lopez Arteaga \\ Department of Mechanical Engineering Dynamics and Control, Eindhoven University of \\ Technology, P.O. Box 513, 5600 MB Eindhoven, The Netherlands
}

a)e-mail: a.aulitto@tue.nl 


\begin{abstract}
The acoustic behavior of individual slits within microslit absorbers (MSAs) is investigated to explore the influence of porosity, edge geometry, slit position and plate thickness. MSAs are plates with arrays of slit-shaped perforations, with the height of the order of the acoustic viscous boundary layer thickness, for optimized viscous dissipation. The slit behaves as confined in a rectangular channel and the flow within the slit is assumed as locally incompressible. The viscous dissipation and the inertia are quantified by the resistive and the inertial end-corrections. These are estimated by using analytical results and numerical solutions of the Linearized Navier-Stokes equations. Expressions for the end-corrections are provided as functions of the ratio of the slit height to the viscous boundary layer thickness (Shear number) and the porosity. The inertial end-correction is sensitive to the far-field behavior of the flow and strongly depends on the porosity, unlike for circular perforations. The resistive end-correction is dominated by the edge geometry of the perforation. The relative position of the slit with respect to the wall of the channel is important for distances of the order of the slit height. The plate thickness does not have a significant effect on the end-corrections.
\end{abstract}




\section{INTRODUCTION}

$1 \quad$ Microslit absorbers and plates (MSAs, MSPs) have been proposed by Maa ${ }^{16}$ as sound absorbers at low frequencies, providing light-weight and compact solutions to substitute conventional materials, such as absorptive foams and porous structures. In simple MSAs 4 the plate, consisting of an array of slit-like perforations, is mounted with a shallow or sub5 partitioned backing cavity. Alternative designs of MSAs have been recently reported in the 6 literature $^{12,19,21,25}$. MSAs have several advantages with respect to micro-perforated plates 7 (MPPs) with circular perforations. Using slits one can easily obtain a relatively large poros-

8 ity, resulting in a higher Helmholtz resonance frequency, when needed. For equal porosity, a

single slit replaces a large number of circular perforations. Furthermore, a slit can be used to delimit flexible structures whose vibration can contribute to the sound absorption ${ }^{21,25}$. Compared to the literature for circular perforations, fewer publications investigate the acoustic properties of slit-like perforations. Maa $^{16}$ states that no theory is available to predict inertial end-correction. He assumes the same viscous dissipation as for circular perforations. In the work of $\mathrm{Maa}^{16}$, radiation to free space is assumed. The inertial end-correction model fails. This failure is solved when taking the confinement into account. Ingard ${ }^{9}$ obtained a solution for high Shear numbers, assuming a uniform flow in the slit and matching the resulting rigid piston oscillation model to a modal expansion of the flow in the confinement channel. Correct expressions for the inertial end-corrections, without typos, are presented by Jaouen et al. ${ }^{10}$. 
The same model is used by Vigran ${ }^{24}$. Another model, based on an incompressible potential flow with a thin boundary layer, is proposed by Morse and Ingard ${ }^{17}$ for an abrupt transition with sharp square edges. This model yields both inertial and resistive end-corrections in the limit of high Shear numbers. For a slit in an infinitely thin plate, the same approach does predict an inertial end-correction. However, the singularity of the potential flow at the edge of an infinitely thin plate results in a divergence of the resistive end-correction. Morse and Ingard ${ }^{17}$ propose as an ad-hoc solution to introduce a finite plate thickness. The divergence of the resistive end-correction due to the singularity at the edge suggests that the viscous dissipation is a local effect, strongly influenced by the edge geometry. One concludes that there is a lack of a complete model to describe the acoustic behavior of slits. For instance, both Ruiz et al. ${ }^{22}$ and Cobo et al. ${ }^{2}$ state that all the models proposed in literature do not fit experimental absorption curves of MSPs. Therefore, the goal of the present work is to complement the theoretical knowledge concerning the acoustical properties of microslits. In particular two effects appear to be ignored in the literature for slits: the influence of the position of the slit and the influence of the edge shape. For a circular perforation, Temiz et al. ${ }^{23}$ observed that chamfering the edges reduces the effective plate thickness $t_{\text {eff }}$ by a length of the order of the total length of the chamfers. A non-symmetric position of the slit can be found when the periodicity of the array is not perfect or in case of sub-partitioned cavity. In the present work, a combination of analytical models and numerical solution of the 
incompressible Linearized-Navier Stokes equations is proposed. In Sec. II, analytical models are developed using a 2D configuration. In Sec. III, the numerical models and solutions of the incompressible Linearized Navier-Stokes equations (LNSE) are proposed using $\mathrm{Comsol}^{3}$ v5.5. In Sec. IV, analytical and numerical results are compared. Findings are summarized in Sec. V.

II. THEORY

\section{A. Definition of the problem}

Microslit plates (MSPs) are plates with arrays of slit-like perforations with height $b$ in the sub-millimeter range and width $w>>b$. The plate thickness $t_{p}$ is of the order of magnitude of the slit height. The acoustic properties of MSPs are defined by the porosity $\Phi=b / a$, with $a$ the distance between neighboring slits. The hydrodynamical interaction between the perforations in the array can be described by considering a single slit of height $b$, confined within a channel of height $a$ of rectangular cross-section aw given by the distance between neighboring slits and the lateral width of the slit. At the open front side of the MSA, the confinement channel represents the hydrodynamic interaction between neighboring slits. 
The confinement channel on the cavity side is resulting from physical walls in case of a sub-partitioned cavity or is due to hydro-dynamical interactions.

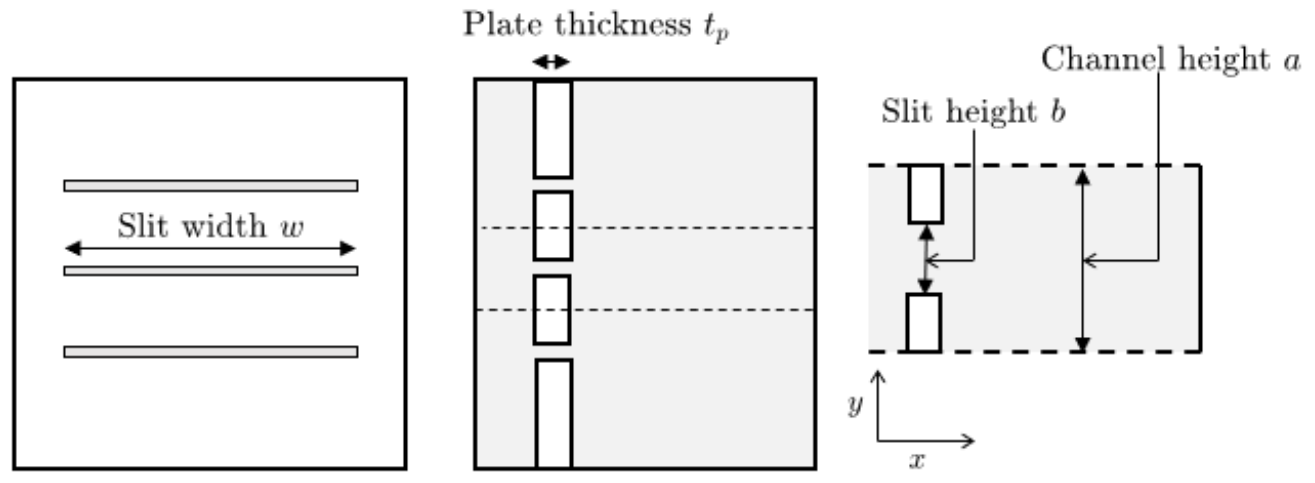

Figure 1: On the left, frontal view of the microslit plate with slit width $w$. In the middle, lateral view of the microslit plate of thickness $t_{p}$ with back cavity. On the right, a single single of height $b$ with confinement channel of height $a$ due to hydrodynamic interactions.

As illustrated in Fig. 1, for a periodic array of slits, the confinement channel is placed

symmetrically with respect to the slit. Assuming a long slit $(w>>b)$ implies that one can consider a two dimensional $(2 D)$ acoustical flow through the slit. As the slit forms the neck of a Helmholtz resonator with a portion of the back cavity as volume, the flow within the slit can be considered as locally incompressible up to the first resonance frequency of the resonator, $\omega_{H}=c \sqrt{\Phi /\left(d_{c} t_{e f f}\right)}$, with $c$ the speed of sound, $d_{c}$ the back cavity depth and $t_{\text {eff }}$ the effective neck length. In the audio range, the square of the Helmholtz number is small,i.e. $H e^{2}=\left(\frac{\omega b}{c}\right)^{2}<10^{-1}$. Thermal effects in the slit are neglected. The discussion is 
limited to the normal incidence of acoustic waves. One can describe the transition between the slit and the confinement channel by assuming over the plate thickness $t_{p}$ an ideal $2 \mathrm{D}$ parallel flow for a long slit of height $b$ extended over a so-called end-correction length. The extrapolation of the linear acoustic pressure in both the slit and the confinement channel is used to define the end-corrections. There is a resistive end-correction $\delta_{\text {res }}$ and an inertial end-correction $\delta_{\text {in }}$ corresponding to the pressure components $\operatorname{Re}[\hat{p}]$ and $\operatorname{Im}[\hat{p}]$, respectively in phase with the volume flow oscillation and in phase with the acceleration of the volume flow oscillation $\widehat{U} e^{i \omega t}$. The inertial end-correction determines the Helmholtz resonance frequency, as shown in Zielinski et al. ${ }^{25}$. Assuming the same geometry on the front and backside of the slit, the effective neck length is given by $t_{e f f}=t_{p}+2 \delta_{i n}$. The resistive end-correction, takes into account the viscous dissipation and influences the quality of the Helmholtz resonance. To optimize viscous dissipation, the slit height is chosen to be of the order of magnitude of a few times the acoustical viscous boundary layer thickness $\delta_{v}=\sqrt{2 \nu / \omega}$, where $\nu$ is the kinematic viscosity of air and $\omega=2 \pi f$, with $f$ the frequency. Hence, for typical applications the Shear number $S h_{b}=b / \delta_{v}$ is of order unity. The range $0.05<S h_{b}<20$ is considered. As the plate thickness and end-corrections in MSPs are both typically of the order of the slit height, it is important to obtain an accurate prediction of end-corrections to design the absorbers. 


\section{B. Parallel flow}

An analytical model for the flow in a long slit of height $b$ is used as a reference to define the end-corrections and to define low and high Shear number limits. It is also used to assess the accuracy of the numerical solution of the incompressible Linearized Navier-Stokes equations. At low Helmholtz numbers $\left(H e^{2}<<1\right)$, in absence of main flow, the acoustic field is described by the equation of continuity

$$
\nabla \cdot \vec{v}=0
$$

and the linearized equation of motion

$$
\rho_{0} \frac{\partial \vec{v}}{\partial t}=-\nabla p+\eta \nabla^{2} \vec{v}
$$

where $\vec{v}$ is the velocity, $p$ is the pressure fluctuation, $\rho_{0}$ density of the air assumed to be uniform and constant and $\eta$ is the dynamic viscosity. In a long thin slit of height $b$, width $w>>b$ and length $t_{p}>>b$, for $0<x<t_{p}$ and $-b / 2<y<b / 2$, the flow can be approximated by a parallel flow $\vec{v}=(u(y, t), 0,0)$. The continuity equation implies, in a parallel flow, that $\frac{\partial u}{\partial x}=0$. Hence, the derivative with respect to $x$ of the $x-$ component of the equation of motion implies that $\frac{\partial^{2} p}{\partial x^{2}}=0$, i.e. the pressure is given by a linear function of the $x$-coordinate. The $y-$ and $z$-components of the equation of motion reduce to $\frac{\partial p}{\partial y}=\frac{\partial p}{\partial z}=0$. This results in a uniform pressure in a cross-section of the slit. Consequently one has that 
101

$\frac{\partial p}{\partial x}=\Delta p / t_{p}$ with $\Delta p=p\left(t_{p}, t\right)-p(0, t)$. For a harmonic oscillation $\Delta p=\Delta \widehat{p} e^{i \omega t}$ the flow profile satisfying the no-slip boundary condition $(u, v)=(0,0)$ on the slit walls $y= \pm b / 2$ is

$$
u(x, t)=\widehat{u} e^{i \omega t}=-\frac{i}{\rho_{0} \omega} \frac{\Delta \widehat{p}}{t_{p}}\left[1-\frac{\cosh \frac{(1+i)}{\delta_{v}} y}{\cosh \frac{(1+i)}{2 \delta_{v}} b}\right] e^{i \omega t} .
$$

The cross-sectional averaged amplitude of the velocity $\langle\widehat{u}\rangle$ is

$$
\begin{aligned}
<\widehat{u}>=\frac{1}{b} \int_{-b / 2}^{b / 2} \widehat{u} d y=-\frac{i}{\rho_{0} \omega} \frac{\Delta \widehat{p}}{t_{p}} & \\
& \cdot\left[1-\frac{2}{(1+i) S h} \tanh \frac{(1+i)}{2} S h\right] e^{i \omega t},
\end{aligned}
$$

with $S h_{b}=b / \delta_{v}$, the Shear number. The slit impedance $Z_{b}$ is defined as in Morse and Ingard ${ }^{17}$ :

$$
Z_{b}=\frac{\Delta \widehat{p}}{w b<\widehat{u}>}
$$

At low Shear numbers $S h_{b}<1$, one can use the approximation

$$
Z_{b} \approx \frac{12 \eta t_{p}}{(w b) b^{2}}+i \frac{6}{5} \rho_{0} \omega t_{p}
$$

One recognizes in the real part of $Z_{b}$ the resistance corresponding to a parabolic flow (quasisteady flow approximation). At high Shear numbers $S h_{b}>>1$, one has

$$
Z_{b} \approx \frac{\rho_{0} \omega t_{p}}{(w b) S h_{b}}+i \frac{\rho_{0} \omega t_{p}}{(w b)}\left(1+\frac{1}{S h_{b}}\right) .
$$

The first part of the imaginary part corresponds to the inertia of a uniform flow, which is a factor 6/10 lower than that of a parabolic flow (see Eq. 6). The time-averaged viscous 
dissipation $\bar{P}_{W}$ in the slit is given by Morse and $\operatorname{Ingard}^{17}$ :

$$
\bar{P}_{W}=\frac{1}{2} \operatorname{Re}\left[Z_{b}\right]|<\widehat{u}>|^{2}(w b)^{2} .
$$

For $S h_{b}>>1$ using Eq. 7 one has

$$
\bar{P}_{W}=\frac{1}{2} \rho_{0} \omega \delta_{v}|<\widehat{u}>|{ }^{2} 2 w t_{p}
$$

This thin boundary layer approximation is used in Sec. II E for channels with non-uniform height. In this limit, the flow in the boundary layer is quasi-parallel along the wall. Therefore, one can use the dissipation per unit surface found in Eq. 9 when replacing $|<\widehat{u}>|^{2}$ by the amplitude of the tangential velocity amplitude $\left|\widehat{u}_{t a n}\right|$ prevailing just outside the viscous boundary layer. Integration over the surface yields the total dissipation. This tangential velocity corresponds to that of a frictionless potential flow. Alternative derivations of this thin boundary layer equation are provided in literature ${ }^{1,14,17,20}$. As explained by Morse and Ingard $^{17}$, this approximation fails for infinitely thin orifice plates. While Morse and Ingard ${ }^{17}$ suggest that the approximation is valid for sharp square edges, the numerical integration of the Linearized Navier-Stokes equations will allow to verify this assumption.

\section{Impedance and end-corrections}


In this subsection a formal definition of impedance and end-corrections is provided. Consider the transition from a slit of height $b$ to a channel of height $a>b$. In an ideal (reference) configuration the transition from the slit to the channel is abrupt: the flow can be described as a piece-wise parallel flow. In the actual flow, the transition from the slit to the channel is smooth. However, far from the transition one can observe the linear change in pressure, corresponding to a parallel flow in a slit of height $b$ and in a confinement channel of slit of height $a$. This far field can be extrapolated at each side of the transition towards the plate surface at $x=0$. The complex difference in pressure $\Delta \widehat{p}_{t}$ obtained across the transition by this extrapolation divided by volume flux amplitude $\widehat{U}=<\widehat{u}>b w$ is defined as the transition impedance $Z_{t}$. The inertial end-correction $\delta_{i n}$ and the resistive end-correction $\delta_{\text {res }}$ are defined by:

$$
\delta_{i n}=\frac{\operatorname{Im}\left[Z_{t}\right]}{\operatorname{Im}\left[\frac{d Z_{b}}{d t_{p}}\right]},
$$

$$
\delta_{\text {res }}=\frac{R e\left[Z_{t}\right]}{\operatorname{Re}\left[\frac{d Z_{b}}{d t_{p}}\right]} .
$$

The resistive end-correction $\delta_{\text {res }}$ is in principle different from the inertial end-correction $\delta_{i n}$. In Fig. 2 several values of the inertial and resistive end-corrections obtained from the literature for perforations with sharp edges are shown as function of the inverse of the plate porosity $1 / \Phi=a / b$. Results for circular perforations are also displayed. A critical discussion of these data is provided by Kergomand and Garcia ${ }^{11}$. 


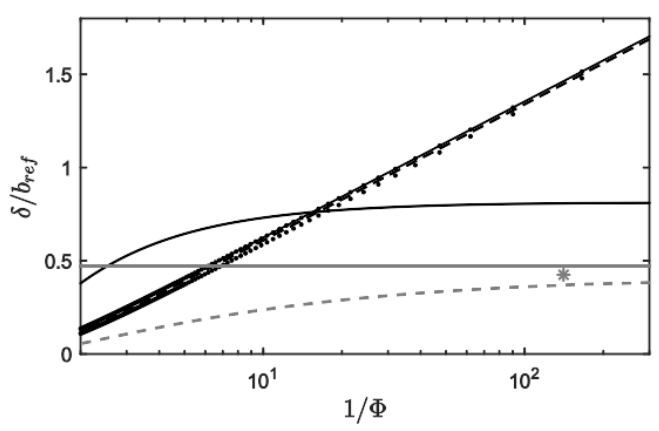

Figure 2: Comparison of end-corrections for sharp-edged slit $\left(L_{r e f}=b\right)$ and circular perforation $\left(L_{r e f}=d_{p}\right)$ from the literature. Inertial end-corrections $\delta_{i n}$ for slits (MSPs): -high $S h_{b}$ number limit of Morse and Ingard ${ }^{17},-.-$ Modal expansion of $\operatorname{Ingard}^{9}, \ldots$ Thin boundary layer of Morse and Ingard ${ }^{17}$. - Slit Resistive end-correction $\delta_{\text {res }}$ from Morse and Ingard ${ }^{17}$. - - - Inertial end-correction for circular perforations from Fok ${ }^{4}$. Resistive endcorrection for circular perforations: $*$ from Temiz et al. ${ }^{23}$ and - from Naderyan et al. ${ }^{18}$.

The reference length $L_{r e f}$, in Fig. 2 refers either to the height $b$ for slits or to the perforation diameter $d_{p}$. It can be noted that the various results at high $S h_{b}$ numbers for the inertial end-corrections for sharp-edged slits are in close agreement. Note that the inertial end-correction for a slit in an infinitely thin plate is quite close to that for a square edge transition from a slit to a higher channel. This indicates that at high Shear numbers the plate thickness has a minor effect on the inertial end-correction. For a slit at low porosity $\Phi=b / a$ it appears that the inertial end-correction is proportional to $\ln (1 / \Phi)$. Hence, it becomes infinitely large for vanishing porosity. This divergence can be removed when taking 
into account the influence of the flow compressibility ${ }^{13}$. For a circular perforation the finite limit value ${ }^{15} \delta_{i n, \Phi \rightarrow 0}=0.4108 d_{p}$ is found for vanishing small porosities. For the resistive end-correction of slits, Morse and Ingard ${ }^{17}$ obtained an analytical result for $S h_{b}>>1$. The resistive end-correction increases with decreasing porosity but reaching an asymptote for $\Phi \rightarrow 0$. For circular perforation, resistive and inertial end-corrections are of the same order of magnitude. It should be noted that for relevant porosities all end-corrections are of the order of $L_{r e f}\left(\right.$ either $b$ or $\left.d_{p}\right)$. derive an expression for the inertial end-correction. Given an arbitrary velocity profile at the end of the slit, it is possible to derive the inertial end-correction by matching to acoustic modes in the confinement channel. Outgoing plane wave and evanescent transversal modes are considered. Kergomand and Garcia ${ }^{11}$ discuss the convergence of the modal expansion. When using the rigid piston approximation in the slit the number of modes used in the channel should be of the order of the inverse of the porosity ${ }^{20} 1 / \Phi=a / b$. An expression of the inertial end-correction for low the $S h_{b}$ number is obtained by assuming a parabolic flow (see Sec. ÏIB) at the end of the slit. This is used as input for the frictionless modal 
expansion of the acoustic pressure in the channel. One finds:

$$
\begin{aligned}
\delta_{i n}=\frac{5}{6} \sum_{n=1}^{\infty} \frac{3}{2 n \pi}\left(\frac{a}{n \pi b}\right)^{3}\left\{4 \cos ^{2}(n \pi)\right. & \\
& \left.\cdot\left[\cos \left(\frac{n \pi b}{a}\right)-\frac{a}{b n \pi} \sin \left(\frac{n \pi b}{a}\right)\right] \sin \left(\frac{n \pi b}{a}\right)\right\} .
\end{aligned}
$$
investigated. In Fig. 3, the transition from an asymmetric slit to a channel is displayed.

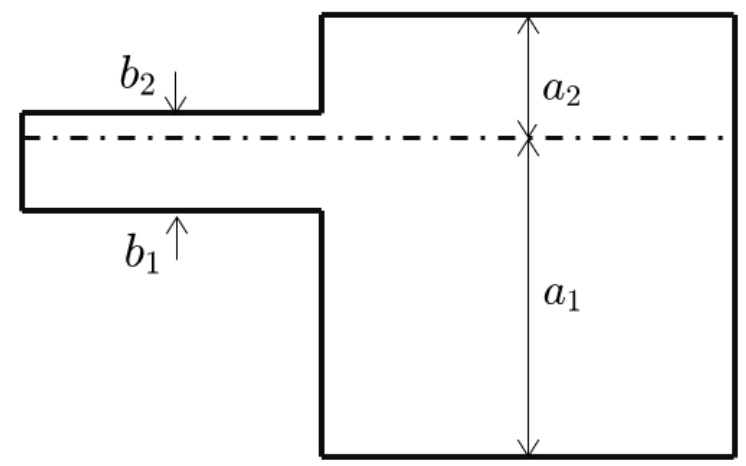
accuracy.

For the asymmetric case, the influence of the position of the slit with respect to the wall is

Figure 3: Geometry of the asymmetric slit of height $b=b_{1}+b_{2}$ emerging in a channel of

height $a=a_{1}+a_{2}$.

163

A number of modes of the order of $N_{m}=3(a / b)$ is sufficient to reach a reasonable

The slit height is $b=b_{1}+b_{2}$, the channel height is $a=a_{1}+a_{2}$. The geometry is chosen such that the $(a / b)=\left(a_{1} / b_{1}\right)=\left(a_{2} / b_{2}\right)$. In the limit case of a slit sharing the flat wall with the channel, one has $a_{2}=0$ or $a_{1}=0$. The vertical positions of the slit edges $($ at $x=0)$ are 
$y_{1}=a_{1}(1-b / a)$ and $y_{2}=a-a_{2}(1-b / a)$. Assuming at the end of the slit a uniform acoustic velocity amplitude and expanding the amplitude of the pressure in frictionless modes in the channel one finds:

$$
\delta_{i n}=\sum_{n=1}^{\infty} \frac{2}{n \pi}\left\{\frac{\left[\sin \left(\frac{n \pi y}{a}\right)\right]_{y_{1}}^{y_{2}}}{\frac{n \pi b}{a}}\right\} .
$$

In the symmetric case $a_{1}=a_{2}$ one finds the result of $\operatorname{Ingard}^{9}$, where $n=2 m$. The influence of the position of the slit on the inertial end-correction is discussed in Sec. IV C.

\section{E. Thin boundary layer approximation}

For the high $S h_{b}$ range, the viscosity effects are concentrated in a thin boundary layer at the wall and do not impact the main, potential flow. The incompressible potential flow theory combined with the thin boundary layer approximation proposed by Morse and In$\operatorname{gard}^{17}$ can be used. A generalization of this model is presented by Berggren et al. ${ }^{1}$. In the present work, this approximation is used to investigate the end-corrections for smooth edges and asymmetric slit sharing the flat wall with the confinement channel. It is also used to explore the effect of viscous friction along the confinement channel walls. The smooth edge geometry is obtained using the conformal transformation introduced by Henrici ${ }^{6}$ (Appendix A for details). An analytical solution is proposed for a smooth transition, providing a generalization of the results of Morse and Ingard ${ }^{17}$ for sharp edges. 


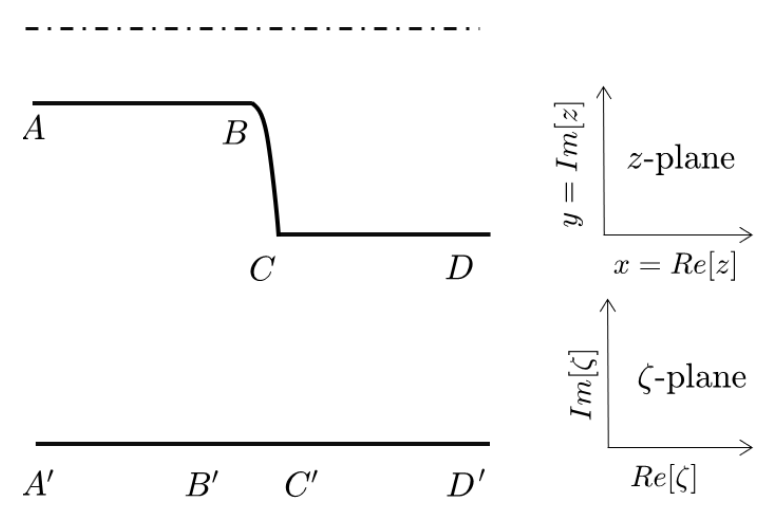

Figure 4: Henrici's transformation of half the channel with smooth transition from the slit to the channel in the physical plane $z=x+i y$ to the $\zeta$-plane. Coordinates of the points: $A(-\infty ;(a+b) / 2), B(-d ;(a-b) / 2), C(0,0), D(\infty ; 0)$.

In Fig. 4, a 2D slit of height $b$ in $x<0$ and a 2D channel of height $a>b$ in $x>0$ are shown. The end of the uniform slit (point B in Fig. 4) is at $(x, y)=(-d, a-b)$, with $d$ being the transition length. The uniform confinement channel begins at $x=0$. The duct can be associated to a region in the complex $z$-plane by $z=x+i y$, with $i^{2}=-1$ and coordinates $(x, y)$. Using conformal mapping, the flow region in the duct can be mapped into the upper half-plane in the complex $\zeta$-plane. The mapping of the contraction is a modified SchwarzChristoffel transformation introduced by Henrici ${ }^{6,}\left({ }^{7}\right)$. The differential form of Henrici's 
transformation is

$$
\frac{d z}{d \zeta}=\zeta^{-1}\left[\alpha(\zeta-1)^{1 / 2}+\beta\left(\zeta-G^{2}\right)^{1 / 2}\right]
$$

$$
\cdot\left(\zeta-G^{2}\right)^{-1 / 2}
$$

where $\alpha, \beta$ and $G$ are parameters of the transformation depending on the slit and channel heights and on the transition length $d$. The parameters $\alpha$ and $\beta$ are functions of the parameter $G$ obtained numerically as the solution of a non-linear equation. Details are discussed in Appendix A. The equation for the sharp square edge transition is recovered for $d=0$. Using the thin boundary layer approximation one can find the real and imaginary part of the impedance of the transition $Z_{t}$ and the inertial and resistive end-corrections. Formulas are provided in Appendix B.

A similar approach can be followed for a fully asymmetric slit, presented in Fig. 3. When $a_{2}=0$, the slit and the confinement channel share the flat wall. One has to add the dissipation of the flat wall, shared by the slit and the channel. This will be done by modifying the limits of integration when calculating the total dissipation along the walls (Appendix B). When the confinement channel walls are representing the influence of hydrodynamic interaction, the flow at the channel walls is frictionless. This can also be taken into account by simply modifying the integration limit when integrating to calculate the dissipated power. Details are in Appendix B. 
209

\section{NUMERICAL MODEL}

\section{A. Uniform channel}

Consider a uniform channel of height $b$ and length $t_{p}$, with $t_{p}>>b$. The $x$-axis goes from $x=0$ to $x=t_{p}$. The $y$-axis extends between $y= \pm b / 2$. As stated in Sec. II A, the low He number approximation is made. The incompressible Linear Navier-Stokes equations for a $2 \mathrm{D}$ domain in a dimensionless form in the frequency domain are hereby presented:

$$
\frac{\partial u^{*}}{\partial x^{*}}+\frac{\partial v^{*}}{\partial y^{*}}=0
$$

$$
i u^{*}=-\frac{\partial p^{*}}{\partial x^{*}}+\frac{1}{2 S h_{b}^{2}}\left(\frac{\partial^{2} u^{*}}{\partial x^{*^{2}}}+\frac{\partial^{2} u^{*}}{\partial y^{*^{2}}}\right) \text {, }
$$

$$
i v^{*}=-\frac{\partial p^{*}}{\partial y^{*}}+\frac{1}{2 S h_{b}^{2}}\left(\frac{\partial^{2} v^{*}}{\partial x^{* 2}}+\frac{\partial^{2} v^{*}}{\partial y^{* 2}}\right) \text {, }
$$

with $x^{*}=x / b$ and $y *=y / b$. The dimensionless velocity $\left(u^{*}, v^{*}\right)$ is $(u / b \omega, v / b \omega)$ and the dimensionless pressure is $p^{*}=p /\left(\rho_{0}(b \omega)^{2}\right)$. These equations are solved using Comsol Multiphysics. At the inlet $\left(x^{*}=0\right)$ and at the outlet $\left(x^{*}=t_{p} / b\right)$ of the domain the uniform pressure values are imposed: respectively, $p_{\text {inlet }}^{*}=1$ and $p_{\text {outlet }}^{*}=0$. At the walls $\left(y^{*}=y / b= \pm 1 / 2\right)$ no-slip boundary conditions, $\left(u^{*}, v^{*}\right)=(0,0)$ prevail. An unstructured mesh of quadratic 
triangular elements is used, with the finest mesh at the walls. The density of elements at the walls depends on the $S h_{b}$ number: the element sizes at the wall are $0.2 / S h_{b}$ or less, in order to accurately capture the viscous boundary layer. For a height ratio $a / b=10$ and $S h_{b}=20$, in the proximity of the edges the maximum element size is $M_{e l} / b=2 \times 10^{-2}$ and the minimum is $m_{e l} / b=7 \times 10^{-4}$. The original mesh chosen for the standard calculations has a total of 13324 total elements, of which 804 are edge elements (at the walls). Several checks are performed to gain insight into the accuracy of the numerical simulations. Firstly, the computational domain length $t_{p}$ is increased to exclude an influence of the boundary conditions. It appears that the quantity $U_{L}=U t_{p} / t_{r e f}$, with $U$ being the flux in a crosssection of the channel and $t_{r e f}=6 * b$, is constant within a relative deviation of $10^{-5}$ for $0.5<t_{p} / t_{r e f}<2$. Secondly, a mesh convergence study is performed and shows convergence to computer accuracy $\left(10^{-13}\right)$. For this study, three additional meshes are used: one coarser and two finer meshes respectively with half, two and four times the basic number of elements at the wall. To compare the results, the cross-sectional average velocity $<\widehat{u}^{*}>=\int_{0}^{b^{*}} \widehat{u}^{*} d y^{*}$ is used. Comsol ${ }^{3}$ performs the integration element-wise using numeric quadrature of the $4^{\text {th }}$ order. The cross-sectional average velocity in the channel obtained with the numerical simulations shows a deviation of $10^{-4}$ from the analytical solution for the parallel flow, discussed in Sec. II B. 


\section{B. Change in cross-section with sharp square edges}

The set of equations $15-17$ is used to study the channel in Fig. 5 presenting at $x^{*}=0$ a sharp square edged transition from a uniform height $b^{*}$ to a uniform height $a^{*}>b^{*}$. The channel extends from $x^{*}=-t_{b}^{*}$ to $x^{*}=t_{a}^{*}$, with $t_{a}^{*}=6 a / b$ and $t_{b}^{*}=t_{a}^{*} / 2$.

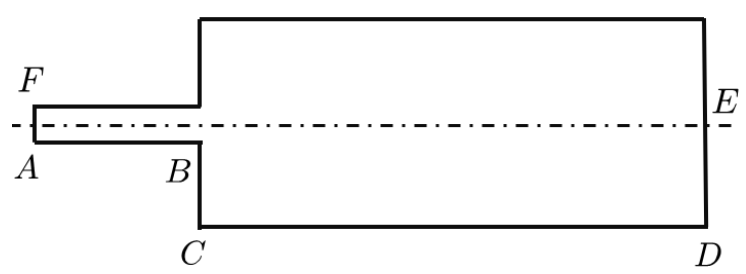

Figure 5: Geometry of a channel with the sudden transition from the slit of height $b$ to the channel of height $a$.

The symmetry of the problem allows limiting the numerical domain to half the channel. For the inlet segment AF and outlet segment DE constant pressures are imposed, $p_{A F}^{*}=1$ and $p_{D E}^{*}=0$. At the segments $\mathrm{AB}$ and $\mathrm{BC}$ the no-slip boundary conditions are applied. At the segment EF (symmetry axis) a slip boundary conditions are implemented: $\partial u^{*} / \partial y^{*}=$ 0 and $v^{*}=0$. The effect of the boundary condition at the lower wall is investigated. When considering a confinement channel due to hydrodynamic interaction, a slip boundary condition is used on the segment CD. Far from the transition located at $x^{*}=0$ the acoustic pressure is uniform in the cross-sectionon and the amplitude of the pressure depends linearly 
on the position along the duct (parallel flow behavior). Assuming that for $-2 a<x^{*}<-1 a$ : $\widehat{p}^{*}(x)=\widehat{A} x^{*}+\widehat{B}$ and for $3 a<x^{*}<5 a$ one has: $\widehat{p}^{*}(x)=\widehat{C} x^{*}+\widehat{D}$. The complex constants can be determined by a linear fit of the pressure data obtained by numerical simulations for these regions far from the discontinuity. The fit gives a coefficient of determination ${ }^{5}$ $1-R^{2}=10^{-6}$. The impedance $Z_{t}$ of the transition is determined by $Z_{t}=\frac{\widehat{B}-\widehat{D}}{\widehat{U}^{*}}$ with $\widehat{U}^{*}$ being the flux calculated in a generic section of the slit far from the discontinuity, defined as $\widehat{U}^{*}=w<\widehat{u}^{*}>b$.

For a porosity $\Phi=b / a=1 / 10$ at $S h_{b}=20$, numerical simulations show that the effect of the boundary condition at the lower wall of the channel is negligible. This is in line with confirming that the dissipation is mainly concentrated inside the slit and around the edges.

In the assumption of locally incompressible flow, the volume flux along the duct axis is constant. This is verified numerically with a maximum relative deviation of $10^{-4}$. The coefficients $\widehat{A}$ and $\widehat{C}$ of the linear fittings of $\widehat{p}^{*}$ can be compared to the theoretical values of the $\Delta \widehat{p}^{*} / t^{*}$ for the parallel flow in a long channel, respectively of height $b$ and $a$. The discrepancy is in the order of $10^{-4}$. Apparently, the accuracy in the calculation of the volume flux is the limiting factor for the global accuracy of the numerical model.

\section{RESULTS}


For $S h_{b}<0.6$, the dimensionless inertial end-correction $\delta_{i n} / b$ and the resistive end-correction $\delta_{\text {res }} / b$ are functions of the porosity and, to a much lesser degree, of the $S h_{b}$ number. The

\subsubsection{Inertial end-correction at low and high $S h_{b}$ number}

In this subsection, the inertial end-correction for a sharp square edged transition derived from the numerical simulations are compared with the analytical solutions proposed in Sec.

II. For low Shear numbers, the inertial end-correction can be calculated using the oscillating parabolic flow approximation. For high Shear numbers the modal expansion of Ingard $^{8}$ and the thin boundary layer approximation of Morse and Ingard $^{17}$ are used. The inertial endcorrection calculated with the parabolic flow approximation is about twice $(5 / 3)$ the value for uniform flow. In Fig. 6 the comparison between the numerical, the modal expansions and thin boundary layer approximation, are shown as function of the inverse of the porosity $\Phi$. The numerical results are obtained for a $S h_{b}=0.05$ and for $S h_{b}=20$. It appears that the parabolic flow approximation captures well the behavior of the inertial end-correction for $S h_{b}=0.05$, whereas the rigid piston and thin boundary layer models are in good agreement with the result for $S h_{b}=20$.

\subsubsection{End-corrections at Low $S h_{b}$ number}

\section{A. Symmetrical slit with sharp square edges}




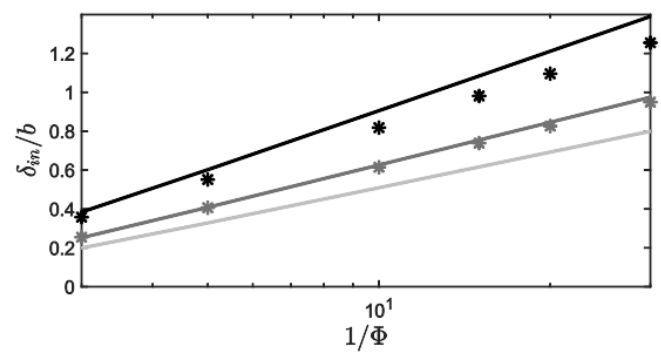

Figure 6: Comparison of inertial end-correction $\delta_{\text {in }}$ as functions of $1 / \Phi$ : - Parabolic flow approximation, - Uniform flow approximation ${ }^{9}$. - High the $S h_{b}$ number limit ${ }^{17}$. Stars refer to the results of numerical calculations for $* S h_{b}=0.05$ and $* S h_{b}=20$.

dependency of the end-corrections on $S h_{b}$ is therefore neglected. The dependency of $\delta_{\text {res }} / b$ on both porosity and the $S h_{b}$ number is negligible. The following fits are proposed:

$$
\begin{aligned}
\frac{\delta_{\text {in }, \text { fit }}}{b} & =-2.17+2.18 *\left(\frac{1}{\Phi}\right)^{0.13}, \\
\frac{\delta_{r e s, f i t}}{b} & =0.425,
\end{aligned}
$$

for $S h_{b}<0.6$ and $3<1 / \Phi<30$. The coefficient of determination ${ }^{5} 1-R^{2}$ for $\delta_{i n} / b$ is 0.997 . The choice of the fit for $\delta_{\text {res }} / b$ results is a maximum underestimation of the actual value of $2.5 \%$ (see Fig. 7). The negligible effect of the porosity of $\delta_{\text {res }} / b$ indicates again that the dissipation is a local effect at the sharp edges. 

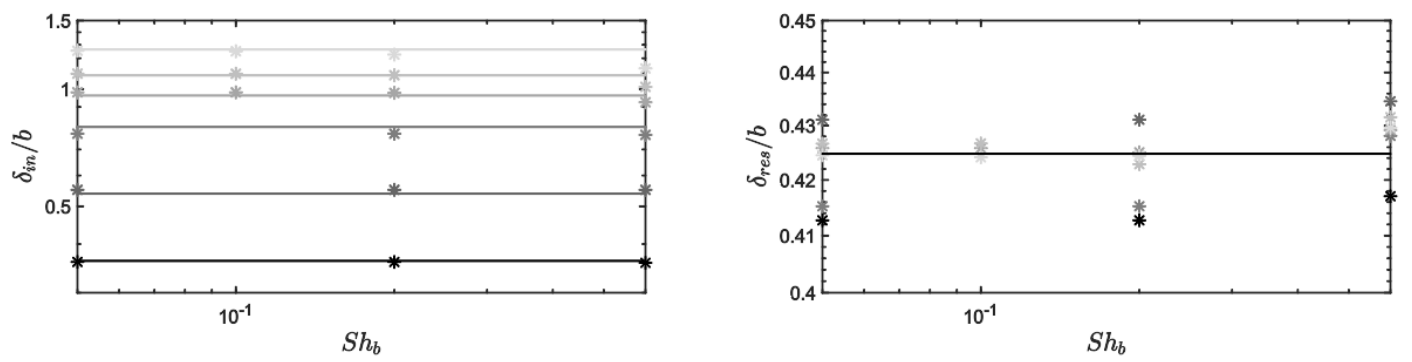

Figure 7: Behavior of a) $\delta_{i n} / b$ and b) $\delta_{r e s} / b$ as function of the $S h_{b}$ number for several porosities for $S h_{b}<0.6:-1 / \Phi=3,-1 / \Phi=5,-1 / \Phi=10,-1 / \Phi=15,-1 / \Phi=20,-$ $1 / \Phi=30$. Solid lines refer to the fit, asterisk refer to numerical data.

\subsubsection{End-corrections at high $S h_{b}$ number}

In the region $0.6<S h_{b}<20$ the deviations of $\delta_{i n}$ and $\delta_{\text {res }}$ from the high $S h_{b}$ limits $\delta_{\text {in,ref }}$ and $\delta_{\text {res,ref }}$, predicted by Morse and Ingard ${ }^{17}$, have been obtained (see Appendix B). Proposed fits of the numerical results are:

$$
\frac{\delta_{i n}}{\delta_{i n, r e f}}-1=\frac{C_{1}}{C_{2}+S h_{b}}
$$

$$
\frac{\delta_{\text {res }}}{\delta_{\text {res,ref }}}-1=\frac{C_{3}}{S h_{b} *\left(C_{4}+S h_{b}\right)} \text {, }
$$

$$
\text { with } C_{i}=D_{i, 1}+D_{i, 2} \cdot(\Phi) \text {. }
$$

From Eq. 22 appears that the coefficients $C_{i}$ are linear functions of the porosity. Table 1 provides the values of the coefficients $D_{i, j}$.

In Fig. 8a and $8 b$ the fits are compared with numerical simulation data. Both the inertial 
Table 1: Values of the coefficients for the fitting in the range $0.6<S h_{b}<20$.

\begin{tabular}{ccccc} 
& $C_{1}$ & $C_{2}$ & $C_{3}$ & $C_{4}$ \\
\hline First coefficient $D_{i, 1}$ & 0.52 & 1.27 & 5.19 & 1.69 \\
Second coefficient $D_{i, 2}$ & 9.34 & 7.45 & 28.74 & 3.97
\end{tabular}

and resistive dimensionless end-corrections show a dependency on the porosity that becomes less important for decreasing porosity. This behavior is more noticeable for $\delta_{\text {res }} / \delta_{\text {res,ref }}$. In

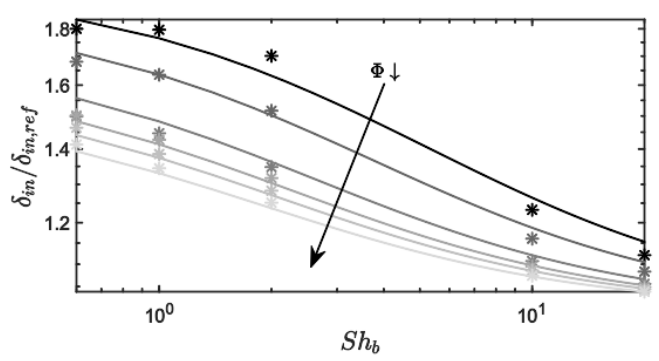

(a)

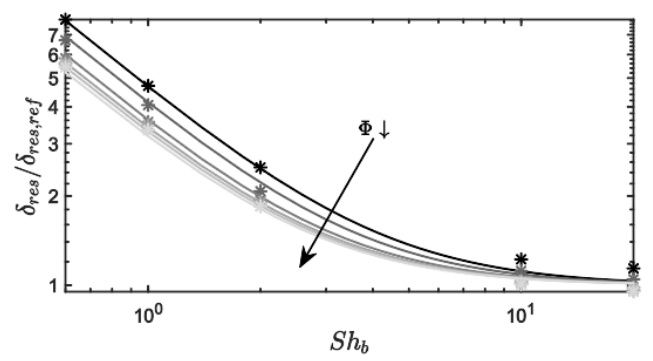

(b)

Figure 8: Result of the fits in the range $0.6<S h_{b}<20$ for a) $\delta_{i n} / \delta_{\text {in,ref }}$ and b) $\delta_{\text {res }} / \delta_{\text {res,ref }}$ as function of the $S h_{b}$ number for several $1 / \Phi=a / b:-1 / \Phi=3,-1 / \Phi=5,-1 / \Phi=$ $10,-1 / \Phi=15,-1 / \Phi=20,-1 / \Phi=30$. Solid lines refer to the fit, asterisk refer to numerical data..

Fig. 9a the linear approximations of the coefficients $C_{1}$ and $C_{2}$ for the inertial end correction are compared with the actual values. In Fig. $9 \mathrm{~b}$ the results for $C_{3}$ and $C_{4}$ for the resistance 
are presented. The average adjusted coefficients of determination ${ }^{5} 1-R^{2}$ are 0.987 for the

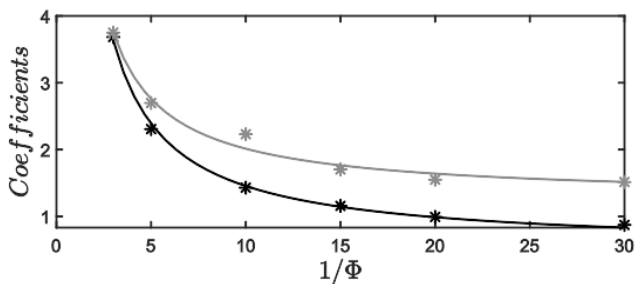

(a)

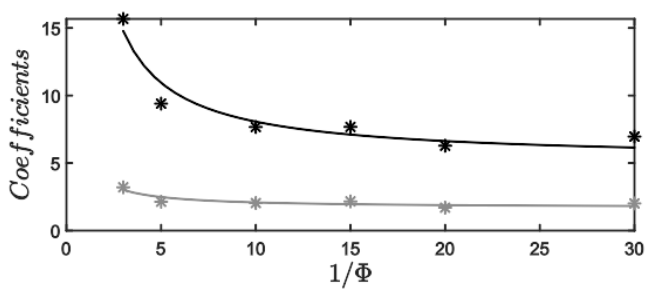

(b)

Figure 9: Comparison of the coefficients $C_{i}$ of the fitting of the inertial and resistive endcorrections as function of the porosity $\Phi$ in the range $0.6<S h_{b}<20$. In a) $-C_{1}$ and $C_{2}$. In b) $-C_{3}$ and $-C_{4}$. In both, asterisks refer to the numerical data and solid lines are referred to the results of the fitting process.

299

inertial term and 0.998 for the resistive term. In Fig. 8a and Fig. 8b it appears that both $\delta_{i n} / \delta_{\text {in,ref }}$ and $\delta_{\text {res }} / \delta_{\text {res,ref }}$ are converging to the unit value for high $S h_{b}$ numbers. For higher $S h_{b}$ numbers, some additional calculations are carried out for a typical porosity $1 / \Phi=10$. At $S h_{b}=100$, one has $\delta_{i n} / \delta_{\text {in,ref }}=1.0116$ and $\delta_{\text {res }} / \delta_{\text {res,ref }}=0.9465$. At $S h_{b}=200$, $\delta_{i n} / \delta_{\text {in,ref }}=1.0061$ and $\delta_{\text {res }} / \delta_{\text {res,ref }}=0.996$. This confirms the validity of the thin boundary layer approximation for sharp square edges. The effect of the boundary condition (slip or no-slip) on the channel walls is investigated for a typical porosity $1 / \Phi=10$ with $S h_{b}=2$ and $S h_{b}=20$. Numerical simulations for $1 / \Phi=10$ show that the introduction of a noslip boundary condition at the walls of the confinement channel has a negligible effect on 
the results. For $S h_{b}=2$, one finds a ratio $\delta_{\text {res,no-slip }} / \delta_{\text {res,slip }}=1.032$. For $S h_{b}=20$, $\delta_{\text {res,no-slip }} / \delta_{\text {res,slip }}=1.044$. Using the thin boundary layer theory, for high $S h_{b}$ one finds $\delta_{\text {res,no-slip }} / \delta_{\text {ressslip }}=1.041$, in agreement with numerical results. One expects that this ratio increases for increasing porosity. For an extremely large porosity $1 / \Phi=3$, one finds a ratio $\delta_{\text {res,no-slip }} / \delta_{\text {res,slip }}=1.185$. One can conclude that the inertial end-correction is determined by the porosity. The porosity has a modest effect on the resistive end-correction. The negligible effect of the no-slip boundary condition in the channel suggests that, for $\Phi=0.1$, dissipation is mainly concentrated around the edges.

\section{B. Symmetric slit with smooth edges}

Consider a slit of height $b$ with rounded edges of radius $r$ placed symmetrically with respect to a channel of height $a$. The results of incompressible LNS simulations are compared to the high the $S h_{b}$ number approximation for a smooth transition discussed in Sec. II E with the shape determined by the transformation of Henrici ${ }^{6}$. Experimental and numerical data for a circular perforation obtained for a $45^{\circ}$ chamfered circular perforation by Temiz et al. ${ }^{23}$ are also displayed. The reference length $L_{r e f}$ is introduced. For the round edges $L_{r e f}=r$, the radius of curvature of the rounded edge. For Henrici's transformation, $L_{r e f}=d$ is the transition length. For chamfered, $L_{r e f}=c_{c h}$ is the chamfer length. It appears that the transition length $d$ well approximates the radius $r$ of an equivalent rounded edge for 
$d / b<1$. In Fig. $10, \delta_{\text {in,round }} / \delta_{\text {in,sharp }}$ and $\delta_{\text {res,round }} / \delta_{\text {res,sharp }}$ are displayed as function of $L_{r e f} / b$. Numerical results for a slit with a height ratio of $a / b=10$ are shown for: 1 ) rounded edges at several $S h_{b}$ numbers $\left.\left(S h_{b}=0.2,2,20\right), 2\right)$ chamfered edges for $c_{c h}=0.5 b$ at $\left.S h_{b}=20,3\right)$ Henrici's geometry for $S h_{b}=20,200$. The analytical potential solution for smooth edges is validated by the LNSE numerical simulations for Henrici's geometry at high $S h_{b}$ number. In Fig. 10a, for the inertial end-correction the analytical solution well approximates the numerical results for a rounded edge. The $2 \mathrm{D}$ planar result for the $45^{\circ}$ chamfered edge is relatively far from the analytical and numerical results for a smooth transition. In Fig. 10b, for the resistive end-correction the analytical solution provides a good approximation for high $S h_{b}$ numbers, both for a round edge and for a chamfered edge. It is interesting to note that the resistive end-correction becomes negative for $L_{r e f} / b$ of order unity. For comparison, the influence of chamfer on circular perforations ${ }^{23}$ is also displayed in Fig. 10. In Fig. $11 \mathrm{a}$ and $11 \mathrm{~b}, \delta_{i n, \text { round }} / \delta_{\text {in,sharp }}$ and $\delta_{\text {res,round }} / \delta_{\text {res,sharp }}$, calculated using the high $S h_{b}$ number limit, are shown for height ratios $a / b$ relevant in MSPs. The inertial end-correction shows a dependencyF on $a / b$ that increases with the increase of the ratio $L_{r e f} / b$. The resistive end-correction shows again a much more modest dependency on the porosity, as already observed for sharp edges. Rounded edges and chamfered edges have a similar effect on the end-correction, for a small radius of curvature of the edge compared to the slit height $b$. The effect of rounded edges on a slit is similar to the effect of a chamfered 


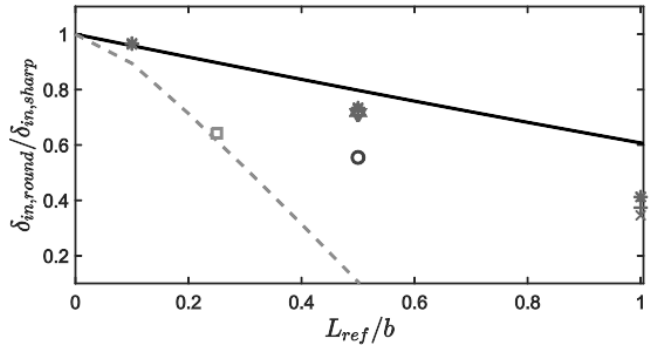

(a)

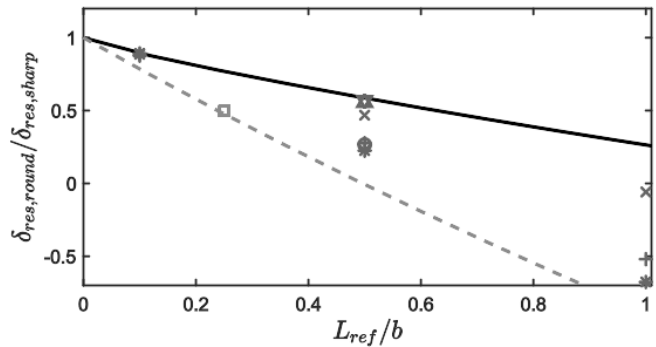

(b)

Figure 10: Comparison of high the $S h_{b}$ number approximation for a smooth transition with numerical results for several ratios $L_{\text {ref }} / b$ for a) $\delta_{\text {in,round }} / \delta_{\text {in,sharp }}$ and b) $\delta_{\text {res,round }} / \delta_{\text {res,sharp }}$ for several $S h_{b}$ numbers: - Slit with smooth transition, $*$ Slit with rounded edges for $S h_{b}=0.2,+$ Slit with rounded edges for $S h_{b}=2, \times$ Slit with rounded edges for $S h_{b}=20, \nabla$ Slit with Henrici's transition for $S h_{b}=20, \triangle$ Henrici's transition for $S h_{b}=200, \bigcirc$ Chamfered edge for $S h_{b}=20,--$ Fit of numerical results and $\square$ Experimental result for circular perforations of Temiz et al. ${ }^{23}$.

\section{Asymmetric slit}




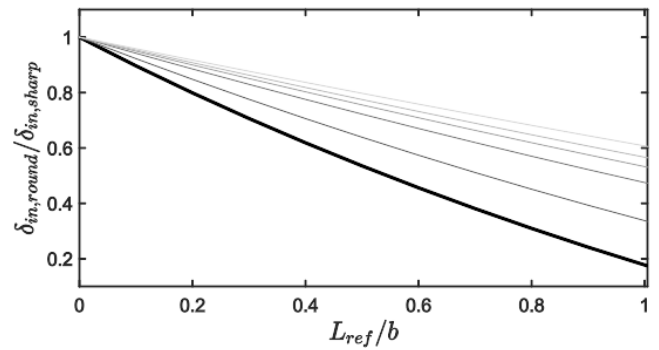

(a)

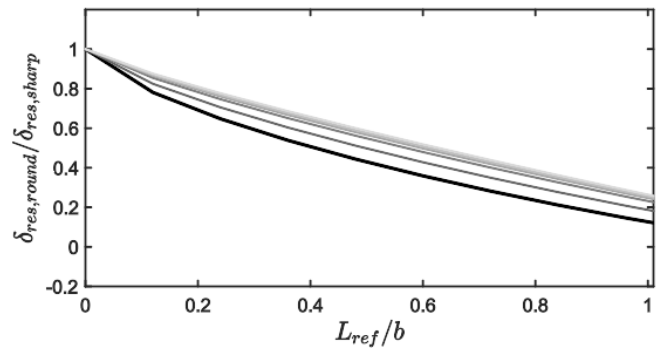

(b)

Figure 11: behavior of a) $\delta_{\text {in,round }} / \delta_{\text {in,sharp }}$ and b) $\delta_{\text {res,round }} / \delta_{\text {res,sharp }}$ as function of the edge rounding $L_{r e f} / b$ for several $1 / \Phi=a / b:-1 / \Phi=3,-1 / \Phi=5,-1 / \Phi=10,-1 / \Phi=15$, $-1 / \Phi=20,-1 / \Phi=30$

In this section results for asymmetric slits are discussed. The position of the slit is determined using the distances $a_{1}$ and $a_{2}$ defined in Fig. 3. The inertial end-correction is calculated for high $S h_{b}$ numbers, using the modal expansion method of Ingard ${ }^{9}$ presented in Sec. II D. In the extreme case that $a_{2}=0$, the high $S h_{b}$ number limit of Morse and Ingard ${ }^{17}$ can be used to calculate both the inertial and the resistive end-corrections. In Fig. 12 the ratio of the inertial end-corrections for the asymmetric case $\left(\delta_{i n, a s y m}\right)$ and the symmetric case $\left(\delta_{i n, s y m}\right)$ is displayed as function of $a_{2} / a_{1}$ for several height ratios $a / b$, with $a=a_{1}+a_{2}$. The value of $a_{2} / a_{1}$ where the effect of the position has a significant effect decreases with the increase of $a / b$. It appears that for a slit positioned at the wall $\left(a_{2}=0\right)$, the inertial end-correction is double the value for the symmetric case, for all the ratios $a / b$. 
Numerical calculations are performed for a slit positioned at the wall and compared to the analytical results. In Fig. 13, for $S h_{b}=20$ the end-corrections for an asymmetric slit $\left(a_{2}=0\right)$ as function of the height ratio $a / b$ are plotted using the corresponding values (same $S h_{b}$ number) for a symmetric slit as a reference. The inertial end-correction is double the value for the symmetric slit. The resistive end correction instead increases for decreasing porosity $\Phi=b / a$. It approaches the asymptotic value of $\delta_{\text {res,asym }}=2.3 \delta_{\text {ress,sym. }}$. This asymptotic value reduces for increasing $S h_{b}$ approaching the analytical value for very high $S h_{b}$ number. Considering the common wall as a mirror, the flow corresponds to that in a slit with double width $2 b$ placed symmetrically with respect to a channel of width $2 a$. This explains the behavior of the inertial end-correction. For the resistive end-correction, the dissipation occurs in a small region around the edge. This region can be addressed as the dissipation region. When keeping the flow velocity in the slit constant, but doubling the slit and channel width, one increases the dissipation region length by a factor 2 . The resulting resistive end-correction doubles. In practice, the end-correction increase is larger than the factor 2 because one has to account for an additional dissipation along the flat wall common to the slit and the channel. While the dissipation is localized around the edge, it is not limited to the edge.

In conclusion, it appears that the influence of the position on the end-corrections cannot be neglected for positions of the slit with respect to the channel of the order of magnitude 


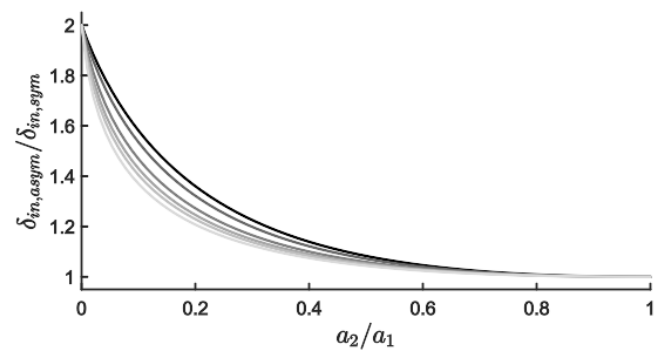

Figure 12: Analytical results for the inertial end-correction obtained by means of modal expansion for an asymmetric slit for several $1 / \Phi:-1 / \Phi=3,-1 / \Phi=5,-1 / \Phi=10,-$ $1 / \Phi=15,-1 / \Phi=20,-1 / \Phi=30$.

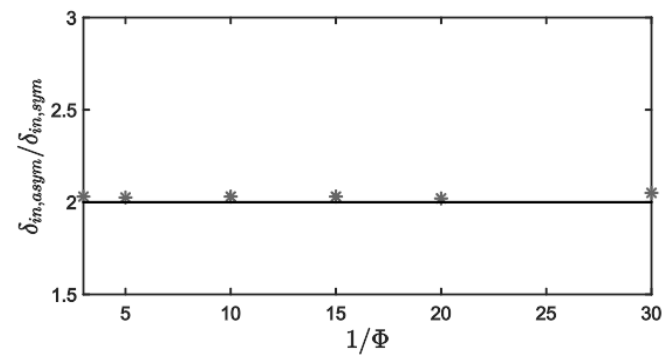

(a)

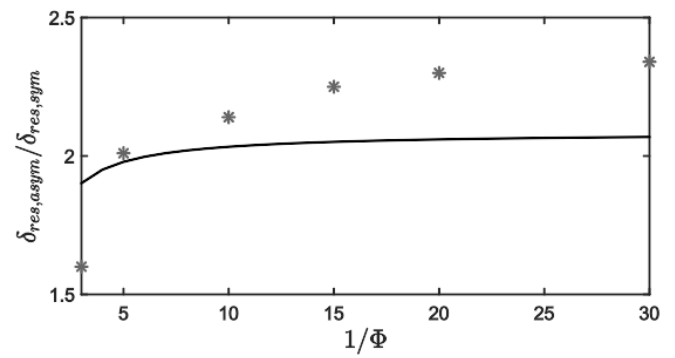

(b)

Figure 13: Comparison of the numerical simulations $(*)$ for $S h_{b}=20$ and potential flow theory (-) results as function of $a / b$ for a) $\delta_{i n, a s y m} / \delta_{i n, s y m}$ and b) $\delta_{\text {res,asym }} / \delta_{\text {res,sym }}$.

of the slit height. nite thickness are compared with the transition between a very long slit and the confinement 


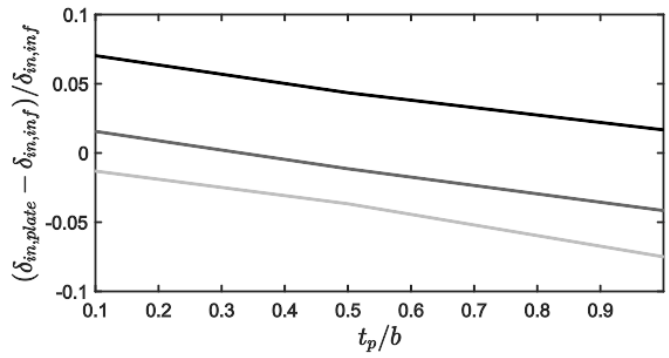

(a) the plate on the end-corrections can be neglected.

\section{1}

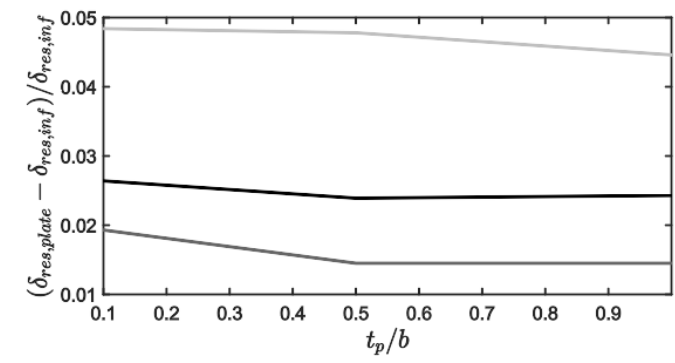

(b)

Figure 14: Deviation of a) $\left(\delta_{\text {in,plate }}\right.$ and b $)\left(\delta_{\text {res,plate }}\right.$ from the semi-infinite slit as function of the ratio $t / b$ for: $-S h_{b}=0.2,-S h_{b}=2$ and $-S h_{b}=20$.

channel discussed in the previous sections. In the range of interest, the deviation lays within $10 \%$ and $5 \%$ accuracy, respectively for the inertial and the resistive end correction. $\delta_{\text {res,plate }}$ shows a negligible dependency on $t_{p} / b$ with respect to the dependency on the $S h_{b}$ number. From this study, one can state that for practical purposes the influence of the thickness of 
to be locally incompressible (low He numbers). Thermal effects are neglected. Focus is given to the frequency range of application for MSAs and resonant metamaterials. For sharp edges, numerical simulations demonstrate that for low $S h_{b}$ numbers a parabolic flow approximation provides a good approximation of the inertial end-correction, whereas the thin boundary layer approximation predicts both the end-corrections at high $S h_{b}$ numbers. The inertial end-correction is strongly dependent on the porosity, showing a very different behavior compared to that of circular perforations. A striking result is that the ratio of the resistive end-correction and the slit height is weakly dependent on the porosity, independently of $S h_{b}$ number. This indicated that the viscous friction is a local phenomenon occurring near the edges. This is confirmed by the negligible influence of the no-slip boundary condition at the walls of the confinement channel, for $\Phi<0.1$. Final prove is gathered in Sec. IV B where the effect of the edge geometry is discussed. The analytical model for a smooth transition provides a reasonable prediction for rounded and chamfered edges at high $S h_{b}$ numbers. In Sec. IV C it is shown that the position of the slit becomes an important effect for distance from the wall in the order of the slit height $b$. For the limit case of a slit sharing the wall with the channel, the inertial and resistive end-corrections are both approximately twice the values for a symmetrical slit. In Sec. IV D it is shown that, for $t_{p}>0.1 b$, the effect of the plate thickness on the end-corrections is negligible. 


\section{ACKOWLEDGMENTS}

The authors acknowledge prof. S. Rienstra for the contributions in conformal mapping and complex function theory. This work is part of the Marie Skłodowska-Curie Initial Training Network Pollution Know-How and Abatement (POLKA). We gratefully acknowledge the financial support from the European Commission under call H2020-MSCA-ITN-2018.

\section{APPENDIX A: TRANSFORMATION OF HENRICI}

In this appendix the high $S h_{b}$ limit theory is described for the smooth and asymmetric transitions presented in Sec. II E. The duct can be associated to a region in the complex $z$-plane by $z=x+i y$, with $i^{2}=-1$ where $(x, y)$ are the coordinates in the physical plane.

Using conformal mapping, the flow region in the duct in the complex $z$-plane is mapped into the upper half-plane in the complex $\zeta$-plane The transformation of Henrici ${ }^{6}$ is used to derive the results for a smooth transition from a slit of height $b$ to a channel of height $a$.

The geometry is presented in Fig. 4. The integral form of the transformation proposed by Henrici $^{6}$ is:

$$
z=\alpha\left[\ln \frac{1+\tau}{1-\tau}-\frac{1}{G} \ln \frac{G+\tau}{G-\tau}\right]+\beta \ln \left[\frac{\zeta}{G^{2}}\right] 7
$$


where $\tau$ is:

$$
\tau=\sqrt{\frac{\zeta-G^{2}}{\zeta-1}}
$$

The point far downstream of the transition $\mathrm{A}\left((-\infty, a)\right.$ can be mapped into point $\mathrm{A}^{\prime}(\zeta=0)$, the start of the transition $\mathrm{B}(-d, a-b / 2)$ corresponds to $\mathrm{B}^{\prime}(\zeta=1)$, the end of the transition $\mathrm{C}(0,0)$ corresponds to $\mathrm{C}^{\prime}\left(\zeta=G^{2}\right)$. The coefficients are related to parameter $\mathrm{G}$ by:

$$
\alpha=\frac{a-b}{\pi}\left[\frac{G}{G-1}\right]
$$

and

$$
\beta=\frac{G b-a}{\pi(G-1)} .
$$

The parameter $\mathrm{G}$ is found by solving the non-linear equation:

$$
G=\frac{a}{b}\left[1+\frac{\pi d}{2 a \ln G}(G-1)\right] .
$$

This equation can be solved by successive substitution for $\pi d(2 b)<2$ using $G_{0}=a / b$ as initial guess. For $\pi d(2 b)>2$ the successive substitutions should be applied to:

$$
G=\exp \left[\frac{\pi d}{2 b}\left(\frac{G-1}{G-\frac{a}{b}}\right)\right],
$$

using $G_{0}=\exp \left(\frac{\pi d}{2 b}\right)$. For sharp edges $d=0$ and $G=a / b$. For an asymmetric slit positioned at the wall it is necessary to identify the point $\zeta_{0}$ on the $\zeta$-axes that corresponds to $z_{0}=i a$ on the flat wall in the $z$-plane. $\zeta_{0}$ is found by solving numerically the equation $z_{0}=z\left(\zeta_{0}\right)=i a$. This can be done for any value of the transition length $d$. Here, only the sharp edge $(d=0)$ is considered for the fully asymmetric slit position $\left(a_{2}=0\right)$. 


\section{APPENDIX B: THIN BOUNDARY LAYER APPROXIMATION}

The thin boundary layer method of Morse and Ingard ${ }^{17}$ for the transition from a slit of height $b$ to a channel $a$ with sharp edges is extended to a smooth transition and to a fully asymmetric slit positioned at the wall $\left(a_{2}=0\right)$. The inertial and resistive end-corrections can be found comparing the actual configuration with an ideal configuration. The ideal reference flow, used to define the end-corrections, has for $x>0$ a uniform velocity $u_{a}$ in the channel of height $a$ and for $x<0$ a uniform velocity $u_{b}=(a / b) u_{a}$. The potential flow far upstream is obtained by placing a volume source at the origin $\zeta=0$ (far downstream the transition) with potential $\varphi=\left(a u_{a} / \pi\right) \ln (\zeta)$. The local flow velocity is the vector field $\vec{v}_{w a l l}=(u, v)=\nabla \varphi$. The linearized form of the frictionless equation of motion is

$$
-\nabla p=\rho_{0} \frac{\partial \vec{v}}{\partial t} .
$$

To compare the actual and the reference configurations two points in the transformed $\zeta$-plane are necessary. Choosing $\zeta_{1} \rightarrow \infty$ and $\zeta_{2}=0$ corresponds to $z_{1}$ and $z_{2}$ respectively far upstream and far downstream the transition. Integrating Eq. 29 between $z_{1}=\left(x_{1} ; y_{1}\right)$ and $z_{2}=\left(x_{2} ; y_{2}\right)$ with $x_{1}>0$ and $x_{2}<0$, one has for a harmonic oscillating acoustic field:

$$
i \rho \omega\left(\varphi_{2}-\varphi_{1}\right)=p_{1}-p_{2},
$$

with $\varphi=\int \vec{v} \cdot d \vec{z}$. If the flow velocity would remain uniform $\left(u_{a}, 0\right)$ for $x>0$ and jump to 
460

$\left(u_{b}, 0\right)$ with $u_{b}=u_{a} a / b$ for $x<0$, we would have:

$$
\left(\varphi_{2}-\varphi_{1}\right)_{i d e a l}=u_{a} \frac{a}{b} x_{2}-u_{a} x_{1}
$$

The inertial end-correction $\delta_{i n}$ is given by:

$$
\delta_{i n}=\frac{u_{a} a}{b \Delta \varphi}
$$

Where $\Delta \varphi$ is defined as the difference $\left(\varphi_{2}-\varphi_{1}\right)_{\text {actual }}-\left(\varphi_{2}-\varphi_{1}\right)_{\text {ideal }}$. Choosing real values $\zeta_{1}$ and $\zeta_{2}$, so that the values of $z_{1}$ and $z_{2}$ are far from the origin of the axis, one has:

$$
\delta_{i n}=\frac{b}{\pi} \ln \left(\frac{\zeta_{2}}{\zeta_{1}}\right)-\operatorname{Re}\left(z_{2}\right) \cdot+\frac{b}{a} \operatorname{Re}\left(z_{1}\right)
$$

For $\zeta_{1} \rightarrow \infty$ and $\zeta_{2} \rightarrow 0$ in Eq. 23 and Eq. 24 we can expand at the first order $\tau$ and obtain an expression for $z_{1}$ and $z_{2}$ to substitute in Eq. 33. One arrives at Eq. 34. For $d=0$ this expression recovers the result of Morse and Ingard $^{17}$.

$$
\begin{aligned}
& \delta_{i n} / b=\frac{1}{\pi}\left\{\frac{(a-b)^{2}}{2 a b} \ln \left(\frac{G+1}{G-1}\right)+\frac{a-b}{b(G-1)}\right. \\
&\left.\cdot\left[\frac{G b+a}{2 a} \ln \left(\frac{(1+G)^{2}}{4 G^{2}}\right)\right]+\ln G\right\} .
\end{aligned}
$$

The additional dissipation due to the transition can be derived integrating along the wall the dissipation per unit surface presented in Sec. II B for the actual and the reference configuration. It should be noted that the actual configuration and the ideal configuration should be combined to obtain converging integrals. In terms of potential the velocity at the wall is:

$$
\left|\widehat{u}_{t a n}\right|^{2}=\left|\frac{d \varphi}{d z}\right|^{2}=\left|\frac{d \varphi}{d \zeta}\right|^{2}\left|\frac{d \zeta}{d z}\right|^{2}
$$


The power dissipated at the junction compared to an ideal configuration is:

$$
\begin{aligned}
2 \vec{P}=\frac{1}{\delta_{v}} \eta w\left[\int_{\zeta_{2}}^{\zeta_{0}}\left(\left|\frac{d \varphi}{d \zeta}\right|^{2} \frac{d \zeta}{d z}-u_{a}^{2} *\left(\frac{a}{b}\right)^{2} \operatorname{Re}\left[\frac{d z}{d \zeta}\right]\right) d \zeta\right. & \\
& \left.+\int_{\zeta_{0}}^{\zeta_{1}}\left(\left|\frac{d \varphi}{d \zeta}\right|^{2} \frac{d \zeta}{d z}-u_{a}^{2} \operatorname{Re}\left[\frac{d z}{d \zeta}\right]\right) d \zeta\right]
\end{aligned}
$$

where for a symmetric slit $\zeta_{1} \rightarrow \infty, \zeta_{2} \rightarrow 0$ and $\zeta_{0}$ corresponds to $z=0$ and it is found from $\zeta_{0}=G^{2}$. The second integral in Eq. 36 contains the effect of the dissipation in the channel. For a slip boundary condition prevailing in a confinement channel resulting from hydrodynamic interactions, one can take $\zeta_{1} \rightarrow \zeta_{0}$ and calculate the dissipation using only the first integral. The resistive end-correction of the discontinuity can be defined as Morse and Ingard ${ }^{17}$ :

$$
\delta_{\text {res }}=\frac{2 \vec{P}}{\left(2 a w u_{a}\right)^{2}} .
$$

Solving the integrals for the symmetric smooth-edged configuration with friction at the channel walls leads to

$$
\begin{array}{r}
\delta_{\text {res }} / b=\frac{(G-1)}{2 G(a-b)}\left\{( G - 1 ) \left[\frac{(G+1}{\pi(G-1)}\left(\frac{(a-b)^{2}}{b^{2}(G+1)}-1\right)\right.\right. \\
\left.\left.\cdot \ln \left(\frac{G+1}{G-1}\right)+1\right]-\frac{2 D G^{2}}{\pi} \ln (G)\right\},
\end{array}
$$

with $D=\frac{G b-a}{G(a-b)}$. Again for $d=0$ one recovers the result of Morse and Ingard ${ }^{17}$, with $G=a / b$ and $D=0$.

For an asymmetric slit, the dissipation of the transition, in this case, is the sum of the dissipation of the edge and the dissipation at the opposite flat wall. The same integrals can 
be solved by changing the integration to $\zeta_{1} \rightarrow \infty, \zeta_{2} \rightarrow-\infty$ and $\zeta_{0}$ can be found solving numerically the equation $z_{0}=z\left(\zeta_{0}\right)=i a$.

\section{REFERENCES}

1. Berggren, M., Bernland, A., and Noreland, D. (2018). "Acoustic boundary layers as boundary conditions," Journal of Computational Physics 371, 633-650.

2. Cobo, P., de la Colina, C., and Simón, F. (2020). "On the modelling of microslit panel absorbers," Applied Acoustics 159, 107118.

3. Comsol (v.5.5.). "Comsol multiphysicsÂA,," COMSOL AB, Stockholm, Sweden. www.comsol.com .

4. Fok, V. (1941). "Teoreticheskoe issledovanie provodimosti kruglogo otverstiya v peregorodke, postavlennoi poperek truby (theoretical study of the conductance of a circular hole in a partition across a tube)," Doklady Akademii Nauk SSSR (Soviet Physics Doklady) 31(9), 875-882.

5. Glantz, S., and Slinker, B. (2001). Primer of Applied Regression 65 Analysis of Variance, ed (McGraw-Hill, Inc., New York).

6. Henrici, P. (1974). Applied and computational complex analysis, Volume I (John Wiley \& Sons). 
7. Hirschberg, L., Schuller, T., Collinet, J., Schram, C., and Hirschberg, A. (2018). "Analytical model for the prediction of pulsations in a cold-gas scale-model of a solid rocket motor," Journal of Sound and Vibration 419, 452-468.

8. Ingård, U. (1948). "On the radiation of sound into a circular tube, with an application to resonators," The Journal of the Acoustical Society of America 20(5), 665-682.

9. Ingard, U. (1953). "On the theory and design of acoustic resonators," The Journal of the acoustical society of America 25(6), 1037-1061.

10. Jaouen, L., and Chevillotte, F. (2018). "Length correction of $2 \mathrm{~d}$ discontinuities or perforations at large wavelengths and for linear acoustics," Acta Acustica united with Acustica 104(2), 243-250.

11. Kergomard, J., and Garcia, A. (1987). "Simple discontinuities in acoustic waveguides at low frequencies: critical analysis and formulae," Journal of Sound and Vibration $\mathbf{1 1 4}(3), 465-479$.

12. Kristiansen, U. R., and Vigran, T. E. (1994). "On the design of resonant absorbers using a slotted plate," Applied Acoustics 43(1), 39-48.

13. Lesser, M., and Lewis, J. (1972). "Applications of matched asymptotic expansion 
methods to acoustics. ii. the open-ended duct," The Journal of the Acoustical Society of America 52(5B), 1406-1410.

14. Lighthill, M. J., and Lighthill, J. (2001). Waves in fluids (Cambridge university press).

15. Maa, D.-Y. (1998). "Potential of microperforated panel absorber," the Journal of the Acoustical Society of America 104(5), 2861-2866.

16. Maa, D.-Y. (2000). "Theory of microslit absorbers," Acta Acustica 25(6), 481-485.

17. Morse, P. M., and Ingard, K. U. (1986). Theoretical acoustics (Princeton University press).

18. Naderyan, V., Raspet, R., Hickey, C. J., and Mohammadi, M. (2019). "Acoustic end corrections for micro-perforated plates," The Journal of the Acoustical Society of America 146(4), EL399-EL404.

19. Randeberg, R. (2002). "Adjustable slitted panel absorber," Acta Acustica united with Acustica 88(4), 507-512.

20. Rienstra, S. W., and Hirschberg, A. (2004). "An introduction to acoustics," Eindhoven University of Technology 18, 19.

21. Ruiz, H., Claeys, C., Deckers, E., and Desmet, W. (2016). "Numerical and experi- 
mental study of the effect of microslits on the normal absorption of structural metamaterials," Mechanical Systems and Signal Processing 70, 904-918.

22. Ruiz, H., Cobo, P., and Jacobsen, F. (2011). "Optimization of multiple-layer microperforated panels by simulated annealing," Applied Acoustics 72(10), 772-776.

23. Temiz, M. A., Lopez Arteaga, I., Efraimsson, G., Åbom, M., and Hirschberg, A. (2015). "The influence of edge geometry on end-correction coefficients in micro perforated plates," The Journal of the Acoustical Society of America 138(6), 3668-3677.

24. Vigran, T. (2014). "The acoustic properties of panels with rectangular apertures," The Journal of the Acoustical Society of America 135(5), 2777-2784.

25. Zieliński, T. G., Chevillotte, F., and Deckers, E. (2019). "Sound absorption of plates with micro-slits backed with air cavities: Analytical estimations, numerical calculations and experimental validations," Applied Acoustics 146, 261-279. 